Tatiana A. Sénina (moniale Kassia)

Saint-Pétersbourg, Russie mon.kassia@gmail.com

\title{
REMARQUES SUR L'AUTEUR ET LA DATE DE SCRIPTOR INCERTUS DE LEONE ARMENIO
}

Il existait quelques hypothèses sur la date et l'origine de la chronique connue sous le titre de Scriptor Incertus de Leone Armenio. ${ }^{1}$ Une confusion supplémentaire a été apportée par la supposition d $\mathrm{d}^{\prime} \mathrm{H}$. Grégoire que Script. Inc. et le récit de la campagne de l'empereur Nicéphore I en l'an $811^{2}$ sont les parties d'une chronique, du prétendu « Continuateur de Malalas $»{ }^{3}$ Cette hypothèse avait été d'abord accueillie avec faveur, mais plus tard quelques objections ont été présentées. Toutefois on continuait de considérer Script. Inc. et De Nicéph. comme deux parties d'une chronique qu'on datait tantôt de la première moitié du $9^{\text {e }}$ siècle, tantôt de son milieu, tantôt de la période après $864 .{ }^{4}$ Cette chronique

(1) Plus loin : Script. Inc. Cette chronique ou plutôt un fragment d'une chronique inconnue a été d'abord édité par I. BEKKER, Leonis Grammatici chronographia, Bonn, 1842, p. 335-362. L'édition critique avec une traduction italienne est parue beaucoup plus tard : F. Iadevaia, Scriptor incertus, Messina, 19972, pp. 25-57 (texte), 67-90 (trad.). A. Markopoulos prépare une nouvelle édition pour la série Corpus Fontium Historiae Byzantinae, mais elle n'est pas encore parue (information de <http://www.oeaw.ac.at/byzanz/cfhb_conspec tus.htm>). Je citerai le texte d'après l'édition d'Iadevaia.

(2) De l'empereur Nicéphore et comment il laissa ses os en Bulgarie (plus loin De Nicéph.), l'editio princeps : И. Дуйчев, « Нови житийни данни за похода Никифора І в България през. 811 г. », Списание на Българската Академия на науките, 54 (1936), pp. 147-188 ; l'édition critique avec la traduction française : I. DujčEv, « La chronique byzantine de l'an 811 », TM, 1 (1965), pp. 205254.

(3) H. GrÉGoIRE, « Un nouveau fragment du "Scriptor incertus de Leone Armenio" ", Byz, 11 (1936), pp. 417-427, ici pp. 417-420; IDEM, Du nouveau sur la chronographie byzantine: le « Scriptor incertus de Leone Armenio » est le dernier continuateur de Malalas, Académie royale de Belgique, Bulletins de la Classe des Lettres et des Sciences Morales et Politiques, 22 (1936), pp. 420-436.

(4) L'histoire du problème voir : Dujčev, "La chronique byzantine... », pp. 207-210, et l'article d'A. Markopoulos (voir la note suivante). Voir aussi : П. Томин, « Фрагменти јудног историског списа IX века », Зборник Радова 
était nommée Scriptor Incertus, et l'édition de F. Iadevaia de 1997 comporte les deux fragments.

Mais la même année A. Kazhdan avec L. Sherry ${ }^{5}$ et A. Markopou$\operatorname{los}^{6}$ ont soumis simultanément à la critique l'avis que Script. Inc. et De Nicéph. avaient paru en réalité sous la plume d'un seul auteur. Leurs arguments, à ce qu'il semble, ont mis le point dans cette discussion : Script. Inc. et De Nicéph., "sous la forme dans laquelle ils nous sont parvenus, sont deux œuvres séparées et sans rapport aussi bien quant au style qu'en ce qui regarde leur structure ».7 Markopoulos signale que le texte de Script. Inc. est « fort hostile à Léon V et, en même temps, nettement favorable au patriarche Nicéphore et au clergé iconophile ", une sorte de pamphlet contre Léon l'Arménien. Le savant estime, en partant du texte de la chronique, qu' «il est fort probable que son auteur ait eu accès aux archives du palais, durant une certaine période au moins, mais surtout aux archives patriarcales ». Il date Script. Inc. de l'époque de Michel II (820-829). ${ }^{8}$

Cependant dans le texte de Script. Inc. on peut trouver quelques raisons de douter d'une datation aussi précoce. Premièrement, c'est la description des souffrances de l'empereur détrôné Michel I Rhangabé :

Michel était un homme tout à fait mûr, ayant passé la fleur de la jeunesse, avec le visage rond des couleurs du froment mûr, aux cheveux crépus sombres et avec une barbe décente, sombre elle aussi, il était très bon et doux comme nul autre. Et il recevait beaucoup de chagrin à cause de la cruauté de l'impie Léon, en attendant la mort tous les jours et en mangeant son pain avec peine chaque jour. Mais le Seigneur l'a préservé miraculeusement sain et sauf des scélératesses de celui-là. ${ }^{9}$

Византолошки Институт, XXI/1 (1952), pp. 78-85 ; R. Browning, « Notes on the "Scriptor incertus de Leone Armenio" », Byz, 35.2 (1965), pp. 389-411, ici pp. 410-411.

(5) A. Kazhdan, L. Sherry, «Some Notes on the Scriptor Incertus de Leone Armenio », Byzantinoslavica, 58.1 (1997), pp. 110-112.

(6) A. Markopoulos, « La chronique de l'an 811 et le Scriptor Incertus de Leone Armenio: problèmes des relations entre l'hagiographie et l'histoire ", RÉB, 57 (1999), pp. 255-262.

(7) Ibid., p. 262.

(8) Ibid., pp. 259, 262.

(9) Script. Inc., p. 40.31-41. 
D'une part, le chroniqueur ne dit rien ici de la mort de Michel mais, d'autre part, le récit de son apparence et de son caractère est fait au passé et le style de la deuxième partie du fragment est presque hagiographique, ce qui peut indiquer indirectement que l'auteur écrivait déjà après le 11 janvier 844 quand le moine Athanase, l'ancien empereur Michel, fut décédé dans le monastère sur l'île Plati. En tout cas notre chroniqueur a manifestement une grande sympathie pour Michel et pour toute sa famille, ${ }^{10}$ et même le fait que ce veule empereur était sous la botte de son épouse ambitieuse (ce sujet est effleuré à peine au début du fragment conservé de Script. Inc.) ne l'indigne point. Il est possible que Script. Inc. utilisait quelques sources proches de la famille de Rhangabé, peut-être venues du patriarche Ignace, fils de Michel. ${ }^{11}$

Deuxièmement, on peût préter l'attention à l'extrait suivant :

Quand la fête était venue, le patriarche s'est adressé à l'empereur avec des paroles d'exhortation, tout en l'appelant à ne pas troubler l'église que les pères avaient rétablie avec une grande peine dans l'orthodoxie après avoir chassé toute hérésie. "Si", a-t-il dit, "c'est à cause de moi qu'a lieu la tentation contre la foi orthodoxe, chassezmoi et arrangez-vous comme vous voudrez, mais n'ébranlez pas la foi !" Et le nommé justement Caméléon a répondu avec feinte et perfidie : "Mais qui osera déposer ou chasser le patriarche et notre père ou bien troubler l'église? Nous n'avons fait qu'un examen à cause des bavards, mais moi je crois comme croit l'eglise". Et, ayant tiré de son sein la croix avec une icône, il l'a adorée devant tout le monde. Pourtant il l'a fait non pas à la vérité mais hypocritement, en voulant attendre la fin de la fête. Car personne ne pouvait réprimer sa perfidie, mais il avait une chose à la bouche et voulait une autre dans son cœur. Toutefois le patriarche et les évêques, ayant entendu cela, se sont réjouis, en pensant qu'il disait la vérité. ${ }^{12}$

À partir du très court récit de Script. Inc. on ne comprend même pas où se passe la conversation entre le patriarche et l'empereur ; on pourrait même penser qu'elle a eu lieu dans une église après l'office et que par « les paroles d'exhortation » on doit entendre le sermon dans

(10) Cf. la description détaillée des bienfaits prodigués par Michel et Procopie, après leur avènement au trône, pour lesquels, comme dit le chroniqueur, «ils étaient glorifiés par tout le monde » (Script. Inc., p. 29.29).

(11) Nicétas avant la tonsure, il a été châtré et forcé à prendre l'habit en 820, avec les autres fils de Michel I, et ménait la vie religieuse sur les îles de Prince ; il a été le patriarche de Constantinople en 847-858 et en 867-877.

(12) Script. Inc., p. 52.426-444. 
lequel le patriarche a appelé publiquement l'empereur à ne pas troubler l'église. En tout cas cet épisode a lieu le jour de la Nativité, c'està-dire après la rencontre des confesseurs orthodoxes avec l'empereur dans le palais le jour de la Nativité du Christ décrite en détail dans les Vies du patriarche Nicéphore ${ }^{13}$ de Théodore le Stoudite ${ }^{14}$ et de Nicétas de Médikion. ${ }^{15}$ Selon la Vie de S. Nicétas de Médikion, vers le jour de la rencontre avec l'impereur les orthodoxes connaissaient déjà " d'une façon exacte l'intention de l'empereur qui s'était plié et se dirigeait vers le mal, et qu'il ne changerait pas d'avis même si l'on lui citait toutes les Écritures pour témoignage $»{ }^{16}$ Mais, chose un peu étrange, l'auteur de Script. Inc., au contraire, dit qu'après la conversation dans le palais le patriarche et les évêques ont cru que l'empereur s'en tenait toujours à l'orthodoxie et s'en sont réjouis. Contre toute attente, un ennemi des iconoclastes, tel que notre auteur, n'a pas dit un mot de la discussion sur l'iconophilie qui s'était déroulée en présence des sénateurs entre l'empereur et les orthodoxes invités par lui au palais et qui a fini pour Léon l'Arménien absolument sans gloire, à l'en croire les sources orthodoxes ci-dessus indiquées.

T. Matantséva a consacré à cette conférence un article particulier. ${ }^{17}$ Elle suppose que la réunion dans le palais avait eu lieu plus tôt, le même décembre 814, mais un peu avant la Nativité, et qu'elle est décrite par Script. Inc. comme l'entretien de Léon l'Arménien avec « les évêques et les higoumènes éminents » envoyés par le patriarche pour répondre aux questions de l'empereur sur les icônes qu'il a posées à

(13) Vie de S. Nicéphore, partriarche de Constantinople (BHG 1335), § 54-58, PG 100, col. 109A-116A.

(14) Vie de S. Théodore le Stoudite : BHG 1755, § 63-75, PG 99, col. 172C$184 \mathrm{C}$, et BHG 1754, §32-34, PG 99, col. 280A-288A.

(15) Vie de S. Nicétas de Médikion, § 32-37; la Vie (BHG 1341), éd. Acta Sanctorum Aprilis I, pp. XXII-XXXII est transmise par un manuscrit unique du début du $X^{\mathrm{e}}$ siècle, sous forme abrégée, surtout vers la fin. Une traduction en slavon permet de restituer le texte original, et je me sers ici de la traduction russe faite par D. Afinogénov, compte tenu des versions greque et slavone : Д. Е. Афиногенов, Житие преподобного отца нашего Константина, что из иудеев. Житие св. исповедника Никить, игумена Мидикийского, Москва, 2001, pp. 95-106; plus loin j'indiquerai les paragraphes du texte.

(16) Vie de S. Nicétas de Médikion, § 34.

(17) T. MatAntséva, « La conférence sur la vénération des images en décembre 814 », RÉB, 56 (1998), pp. 249-260. 
Nicéphore en tête-à-tête ;18 après celà les iconophiles ont fait la veillée en priant Dieu de garder l'Église sans ébranlement, ce qui a irrité l'empereur et l'a provoqué à enlever l'icône du Christ de Chalké. Bien que Script. Inc. dise, à l'opposé des Vies précitées, que le patriarche n'a pas pris part à la discussion du clergé avec l'empereur et a appris ses résultats d'après le récit des évêques, T. Matantséva trouve cette divergeance " d'ordre secondaire » et croit qu'il n'y a eu qu'une seule conférence de Léon l'Arménien avec les iconophiles et qu'elle doit être datée du temps avant la Nativité ; la réunion des iconophiles et la lecture du florilège iconoclaste dans la résidence du patriarche ont eu lieu la veille de la fête, et le jour de la Nativité le patriarche a seulement appelé l'empereur à ne pas troubler l'église.

Cependant dans cette démonstration on voit, à première vue, une absence de coordination. C'est le patriarche qui est présenté par Script. Inc. comme le personnage principal du parti des orthodoxes, alors que les noms des autres iconophiles ne sont même pas cités. Comment le chroniqueur pourrait-il "écarter » Nicéphore de la participation à la discussion publique sur les icônes qui avait eu lieu en présence des courtisans et s'était terminée, à en croire les Vies, par le triomphe du patriarche ? À cet égard l'exposé des mêmes événements fait par D. Athinogénov ${ }^{19}$ semble plus vraisemblable : selon lui, la provocation avec l'élimination de l'icône du Christ de la porte de Chalké était une conséquence de l'échec des tentatives de l'empereur de gagner à sa cause le clergé envoyé par le patriarche ; le concile convoqué par Nicéphore la veille de la Nativité et la réfutation du florilège iconoclaste étaient une réponse à cette provocation ; c'est après cette réunion des iconophiles que l'empereur a appelé au palais tous ses participants qui avaient convenu déjà à ce moment du plan d'action envers les iconoclastes.

De l'autre côté, le silence de Script. Inc. sur la participation du patriarche à la conférence des orthodoxes avec l'empereur et sur le triomphe des iconophiles pendant les débats semble correspondre à l'humeur pessimiste de cet auteur. À cet égard on peut aussi mentionner la proposition faite par le patriarche, selon Script. Inc., de le priver de sa chaire si c'est à cause de lui qu'a lieu la tentation du peuple, mais

(18) Script. Inc., p. 50.356-376.

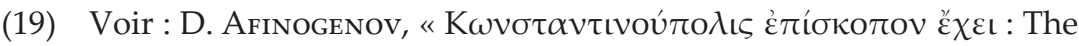
Rise of the Patriarchal Power in Byzantium from Nicaenum II to Epanagoga. Part II : From the Second Outbreak of Iconoclasm to the Death of Patriarch Methodios (847) », Erytheia, 17 (1996), pp. 43-71, ici pp. 48-50. 
de ne pas ébranler la foi. Une telle proposition a le relent de la faiblesse, mais D. Afinogénov le considère comme « nothing but a show of Nicephoros' tactical brilliance $» .{ }^{20}$ C'est possible, mais il est à noter que dans la description de Script. Inc. le patriarche Nicéphore a souvent l'air plutôt d'une victime que d'un diplomate habile et du chef de la résistance orthodoxe comme il se présente dans l'interprétation d'Afinogénov. ${ }^{21}$ En effet, malgré les efforts du patriarche envers l'union de l'opposition orthodoxe décrits par Script. Inc., le sort de Nicéphore est présenté plutôt d'une manière pessimiste : bien que le patriarche eût forcé les évêques et les higoumènes à promettre sous la foi du serment de mourir pour la foi, bientôt ils " ont tourné presque tous à l'inverse », ayant été séduits par les promesses de l'empereur ou ayant eu peur de ses menaces ; le patriarche lui-même est tombé malade et a failli mourir, puis il a failli être tué par une foule excitée, et enfin on l'a envoyé en exil, - tout ce récit ${ }^{22}$ donne l'impression que Nicéphore est resté presque tout seul, abandonné et trahi par tous.

Certainement tout ce qui précède n'est pas un prétexte de penser que Script. Inc. ait été écrit trop de temps après les évenement, - le chroniqueur pouvait tout simplement ne pas être au courant de ce qui s'était passé pendant la conférence au palais, quelle que fut la date de la rencontre, le jour de la Nativité ou quelque peu avant. Cet auteur étaitil trop émoigné du milieu de la création les Vies des confesseurs de l'iconophilie? Ou bien, au contraire, était-il mieux informé des faits et les a-t-il exposés sans embellissements et héroïsme hagiographiques?

Le dernier cas témoignerait que ce texte a été créé en effet à chaud, peu de temps après les évenements, et le ton pessimiste de Script. Inc. peut aussi confirmer cette supposition. Malgré le fait que Léon l'Arménien ait finalement été tué, la description des affaires ecclésiastiques a

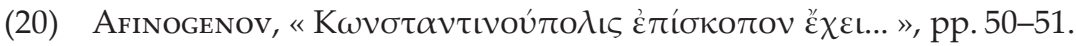

(21) Voir Ibid., pp. 47-57. La conclusion d'Afinogénov que le patriarche Nicéphore, en vue du triomphe prochain de l'iconophilie en 843 , «a gagné sur tous les points » (comme il le dit dans son livre traitant les mêmes problèmes que l'article cité ci-dessus : Д. Е. Афиногенов, Константинопольский Патриархат и иконоборческий кризис в Византии (784-847), Москва, 1997, р. 74) me semble quelque peu hâtive. J'ai déjà porté l'attention à cela dans un autre travail : T. A. Сенина (монахиня Кассия), «Дев Преступник». Царствование императора Аьва V Армянина в отражении византийских хронистов IX века: Феофан Исповедник, Неизвестный хронист (Scriptor Incertus), Георгий Монах (Амартол), Montreal, 2012, pp. 20-22, n. 46.

(22) Script. Inc., pp. 51-55.407-516. 
l'air assez sombre : l'auteur mentionne que «presque tous » les orthodoxes avaient été détournés à l'iconoclasme, décrit les persécutions contre les iconophiles mais ne dit rien sur la résistance orthodoxe ou sur l'héroïsme des confesseurs. Un pareil pessimisme pourrait plutôt saisir un homme qui écrivait pendant le règne de Michel II ou de Théophile, que celui qui travaillait après le rétablissement des icônes. En tout cas nous avons ici un prétexte de refléchir de quel milieu descendait l'auteur de Script. Inc. et en quelle humeur il écrivait son œuvre.

Troisièmement, on doit prêter l'attention au surnom Hylila ( $\Upsilon \lambda\lrcorner \lambda \tilde{\alpha} \varsigma)$ donné par les iconophiles à Jean le Grammairien, le fameux « chef de l'impiété » ( $\left.\alpha \sigma \varepsilon \beta \alpha ́ \alpha \chi \eta \varsigma^{23}\right)$ et l'idéologue principal du second iconoclasme. En cherchant à éclaircir l'origine de ce surnom, B. Lourié a montré24 qu'Hylilas provient du mot hébreu hālāl (qui correspond dans la Septante à $\beta \varepsilon \hat{\varepsilon} \beta \eta \lambda$ os 'vil') et fait allusion à Ezekiel 21, 30-32 où il s'agit de l'impie « prince d'Israël » à qui Dieu ordonne d'enléver la tiare appartenant au Grand Prêtre :

Quant à toi, vil criminel, prince d'Israël dont le jour approche avec le dernier des crimes, ainsi parle le Seigneur Yahvé : On ôtera la tiare, on enlèvera la couronne, tout sera transformé, ce qui est bas sera élevé, ce qui est élevé sera abaissé. Ruine, ruine, ruine, voilà ce que j'en ferai, comme il n'y en eut pas avant que vienne celui à qui appartient le jugement et à qui je le remettrai.

On peut voir ici une allusion à l'illégitimité du patriarchat de Jean, suite à l'injustice et à la violence des empereurs iconoclastes. En effet, Jean était le dernier patriarche iconoclaste, après quoi l'on a mis un terme à cette hérésie et la dignité de patriarche est passée à celui qui devait l'avoir - au patriarche orthodoxe S. Méthode. Dans ce contexte on aurait pu donner à Jean le surnom Hylilas déjà après la restoration des icônes en 843, ce qui reculerait de beaucoup la date de Script. Inc. Mais à ce qu'il paraît, les iconophiles n'ont pas emprunté ce surnom au texte hébreu de la Bible mais l'ont tiré d'une œuvre apocryphe, ${ }^{25}$ et il

(23) Jean est nommé ainsi dans quelques lettres de S. Théodore le Stoudite (voir Theodori Studitae Epistulae, ed. G. Fatouros, Berlin, New-York, 1992, vol. 2, Ep. 332.16, 426.17).

(24) B. LOURIÉ, " Le second iconoclasme en recherche de la vraie doctrine », SP, 34 (2001), pp. 145-169, voir Annexe II, pp. 168-169.

(25) B. Lourié pense que c'était « une œuvre eschatologique perdue remontant (entièrement ou seulement dans ses lignes générales) à un milieu pré-chrétien proche à celui du Targum d'Isaie » (Ibid., p. 169). L'autre sobri- 
est impossible dire dans quel contexte ce surnom y était employé. Il est tout à fait possible qu'Hylilas se présentait dans l'apocryphe vraiment comme un «précurseur et collaborateur du diable », selon l'interprétation de Script. Inc.

Quatrièmement, on peut préter l'attention au passage de Script. Inc. où l'auteur parle du dénigrement subi par le patriarche Nicéphore quand la foule des iconoclastes s'était accumul ée devant la patriarchie :

Et s'étant rassemblés, comme les Juifs contre le Christ, armés de glaives et de bâtons ${ }^{26}$ ils ont envahi la cour de l'eglise en hurlant et chahutant sans frein. Et ils se sont mis à maudire et anathéma-

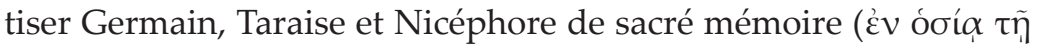

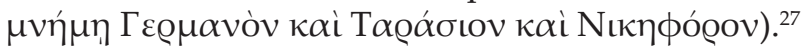

Bien que l'auteur raconte des invectives contre le patriarche Nicéphore encore vivant, la dernière phrase où son nom n'est point séparé

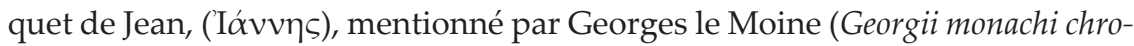
nicon, ed. C. DE Boor, Leipzig, 1904, p. 778.12), remonte aussi à une source apocryphe et a été donné à Jean déjà au début du second iconoclasme, bien qu'on l'ait lié avec l'apocryphe plus tardif. Les noms des mages Jannès et Jambrès qui « se dressèrent contre Moïse » sont devenus communs pour les byzantins comme les noms de ceux qui « se dressent contre la vérité, hommes à l'esprit corrompu, sans garantie en matière de foi » $(2 \operatorname{Tim} 3,8)$. Le surnom Jannès a été inventé possiblement par Théodore le Stoudite qui dans une lettre de 816 appelle Jean « le nouveau Jannès » (Theodori Studitae Epistulae, Ep. 151 : 45), et c'est la première mention de ce sobriquet dans les sources. En tenant compte qu'avant le recommencement de l'iconoclasme Théodore, à juger de ses lettres à Jean, avait été bien disposé envers le Grammairien et l'avait nommé " ami très savant » et avait loué sa sagesse (l'analyse de ces trois lettres voir V. GRUMEL, Jean Grammaticos et saint Théodore Studite, Échos d'Orient, 36 (1937), pp. 181-189 ; leur texte voir Theodori Studitae Epistulae, Ep. 492, 528, 546), il est peu probable qu'en donnant le sobriquet Jannès le Stoudite avait en vue quelque chose d'autre que « l'esprit corrompu » par l'hérésie. Toutefois plus tard les iconophiles commencent à accuser Jean de sorcellerie et de magie (notons que ces accusations ne figurent pas dans les lettres de Théodore le Stoudite). Il est possible que ces inculpations aient pris naissance du développement du sujet du " nouveau Jannès » sur la base du livre apocryphe Jannès et Jambrès parlant de ces mages et connu des iconophiles (voir S. Gero, «Jannes and Jambres in the Vita Stephani Iunioris (BHG 1666) », AB, 113 (1995), pp. 281-292, surtout 284-288). Il est intéressant que Georges le Moine ne mentionne pas le sobriquet Hylilas.

(26) Cf. Mat 26, 47; Mar 14, 48

(27) Script. Inc., p. 54.483-488. 
des noms des ses prédécesseurs « de sacrée mémoire » qui avaient défendu l'iconophilie peut témoigner que Script. Inc. était écrit déjà après la mort de $S$. Nicéphore, c'est-à-dire après 828.

C. Mango suppose que Script. Inc. était à la portée des lecteurs dès les années cinquante du $10^{\mathrm{e}}$ siècle et a été utilisé par le moine Saba dans la Vie de S. Joannice le Grand écrite entre 847 et $860 .{ }^{28}$ La date des années $850^{\mathrm{e}}$ pour Script. Inc. concorde entre autres avec ma supposition que la chronique pouvait être écrite après la mort de Michel Rhangabé, c'est-à-dire après 844 . Si l'auteur était en effet lié avec la famille de Rhangabé, il avait dans les années $840^{\circ}$ certaines raisons pour l'humeur pessimiste visible dans Script. Inc. Tout d'abord c'est le conflit extrêmement aigu entre le patriarche Méthode et les Stoudites : ceux-ci ont refusé d'anathématiser certaines œuvres de S. Théodore le Stoudite ${ }^{29}$ très respecté par Michel Rhangabé comme on le sait. À en juger sur la base des sources conservées, le patriarcat de Méthode était marqué d'une forte confrontation non pas entre les iconophiles et les iconoclastes mais déjà parmi les iconophiles eux-mêmes : les confesseurs qui avaient subi les persécutions étaient fâchés que Méthode eût accédé à la demande de l'impératrice Théodora de prier pour son mari décédé Théophile ; la plupart des orthodoxes ${ }^{30}$ étaient bien sûr mécontents de

(28) C. Mango, "The Two Lives of St. Ioannikios and the Bulgarians ", Harvard Ukrainian Studies, VII (1983), pp. 399-400.

(29) Voir en détail : E. von Doвschüтz, « Methodios und die Studiten. Strömungen und Gegenströmungen in der Hagiographie des 9. Jahrhunderts ",

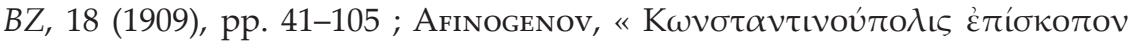
ع̌ $\chi \varepsilon$ L... », pp. 62-70. Toutefois je ne suis pas d'accord avec certaines interprétations d'Afinogénov et surtout avec son attitude négative envers les actions des Stoudites. Méthode visait à détruire les œuvres de Théodore écrites pendant la polémique ardente à propos du deuxième mariage de l'empereur Constantin VI et contenant une critique violente des opposants, y compris des patriarches Taraise et Nicéphore.

(30) N'oublions pas que depuis peu une partie considérable de ces orthodoxes avait été en communion avec les iconoclastes, y compris presque toute l'élite laïque de la Byzance en commençant par ceux qui sont devenus régents sous le mineur Michel III : même si quelquesuns d'entre eux avaient été iconophiles en secret, ils avaient tous communié avec les iconoclastes et avaient pris part dans toutes les cérémonies religieuses conduites par les iconoclastes, sinon ils n'auraient pas pu rester au service de la cour. Entre autres, le logothète Théoktistos connu comme un des initiateurs de la restauration de l'orthodoxie en 843, douze ans auparavant était non seulement un iconoclaste convaincu, mais l'un de ceux qui avaient fouetté le confesseur orthodoxe 
l'attitude sévère du patriarche envers le clergé orthodoxe ; ${ }^{31}$ les orthodoxes influencés par les Stoudites, assez nombreux en tenant compte de la grande autorité de Théodore de son vivant comme après sa mort, ne pouvaient certainement pas être ravis de ce qu'aucun d'entre les Stoudites n'avait reçu une chaire épiscopale sous le nouveau patriarche et que celui-ci les avait même excommuniés et avait jeté l'anathème contre eux pour leur refus de détruire les œuvres de Théodore. Bref, la situation était vraiment déprimante : pendant les persécutions les orthodoxes avaient cherché à oublier les dissentiments d'autrefois et à défendre ensemble l'iconophilie, ${ }^{32}$ mais après la victoire ils avait commencé à lutter les uns contre les autres ce qui pouvait décourager beaucoup de fidèles. Les admirateurs de S. Théodore le Stoudite et de ses élèves devaient être particulièrement indignés et peut-être déprimés à cause des actions du patriarche Méthode. Certainement parmi ces mécontents était le successeur de Méthode et fils de l'empereur Michel Rhangabé : étant devenu patriarche en 847, Ignace a annulé toutes les interdictions posées par Méthode aux Stoudites.

Ainsi, on peut admettre que l'auteur de Script. Inc., s'il écrivait en effet après 843, appartenait aux milieux liés avec la famille de Rhangabé et notamment avec le futur patriarche Ignace. Il était assez bien informé des événements qui avaient eu lieu à la cour de Léon l'Arménien, il était bien disposé envers le patriarche Nicéphore et traitait avec scepticisme la plupart du clergé capable d'oublier vite ses serments et de passer aux hérétiques ;33 il est possible que l'auteur ait été laïque. Il

S. Euthyme de Sardes et, au fond, l'avaient fait mourir sous le fouet ; voir T. A. SÉNINA (moniale Kassia), « Un saint fouette un autre : Théoktistos le logothète et Euthyme de Sardes », Scr, 5 (2009), pp. 391-393.

(31) J'accèpte l'interprétation de D. Afinogénov selon laquelle Méthode a déposé tout le clergé iconoclaste sans exception, voir en détail : D. AfinogeNov, "The Great Purge of 843: a Re-Examination », dans : $\Lambda E I M \Omega N$. Studies presented to Lennart Rydén on His Sixty-Fifth Birthday, ed. J. O. Rosenquist, Uppsala, 1996, pp. 79-91.

(32) Théodore le Stoudite en parle tout à fait explicitement dans une lettre à Pierre, métropolite de Nicée, écrite en 823 ; voir Theodori Studitae Epistulae, vol. 2, Ep. 475.22-42, cf. aussi Ep. 269.

(33) Il est à noter que l'on peut trouver des motifs semblables dans les lettres de Théodore le Stoudite qui se plaint maintes fois de la chute des passants aux iconoclastes (voir Ibid., Ep. 222-223) et écrit dans une lettre au prêtre Grégoire que son destinataire était le seul à Constantinople qui n'avait pas eu peur des menaces et était resté fidèle à l'iconophilie (Ibid., Ep. 163). 
a du écrire son œuvre entre 844 et 847 , c'est-à-dire simultanément avec Géorges le Moine. Ceci pourrait expliquer le fait que Script. Inc. soit absent parmi les sources de Géorges.

\section{SUMMARY}

Judging from several passages of the Scriptor Incertus de Leone Armenio one has to admit that the author of this chronicle was working between 844 and 847 and belonged to a milieu connected with the Rhangabe family and especially with the future Patriarch Ignatius. 